\title{
Integration of a Personalized Molecular Targeted Therapy into the Multimodal Treatment of Refractory Childhood Embryonal Tumor with Multilayered Rosettes (ETMR)
}

\author{
Lisa L.R. Hartman $n^{a, b}$ Derrick M. Oaxaca ${ }^{a, c}$ Benjamin Carcamo ${ }^{a, b}$ \\ Harry L. Wilson ${ }^{a, b}$ Jeremy A. Ross ${ }^{c} \quad$ Elisa Robles-Escajeda ${ }^{c}$ \\ Robert A. Kirken ${ }^{c}$

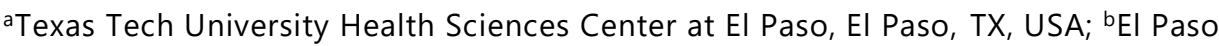 \\ Children's Hospital, El Paso, TX, USA; 'Department of Biological Sciences, The University \\ of Texas at El Paso, El Paso, TX, USA
}

\section{Keywords}

Brain tumor $\cdot$ Pediatric $\cdot$ Targeted therapy $\cdot$ Tyrosine-kinase inhibitor

\begin{abstract}
Embryonal tumor with multilayered rosettes (ETMR) are rare pediatric brain tumors with increased malignant potential. Despite the advances in multimodal treatment schemes the overall 5-year event free survival rates for ETMR are not favorable. Further, therapeutic regimes are limited to a case by case basis due to the limited amount of literature and guidelines available for treating childhood ETMR. We report one patient with refractory ETMR who was successfully treated by implementing a molecular profiling approach which identified the tyrosine kinase inhibitor dasatinib as a viable therapy. Our results suggest that utilizing this precision medicine approach might prove useful in treating patients with refractory ETMR.
\end{abstract}




\section{Case Reports in Oncology}

Case Rep Oncol 2019;12:211-217

\begin{tabular}{l|l}
\hline DOI: $10.1159 / 000497380$ & (c) 2019 The Author(s). Published by S. Karger AG, Basel
\end{tabular} www.karger.com/cro

Hartman et al.: Personalized Molecular Targeted Therapy of ETMR

\section{Introduction}

ETMR (2016 WHO classification) are highly-malignant, and rare, brain tumors that primarily affect young children $[1,2]$. Despite multimodal treatments that include surgery, radiotherapy, and chemotherapy, 3- and 5-year event free survival rates are approximately 34$57 \%$ and $19-38 \%$, respectively $[3,4]$. Moreover, neurocognitive impairment with current radiotherapy treatment strategies is a significant complication for survivors [5]. Implementing molecular biomarkers in the modulation of treatment strategies has proven promising for selectively treating ETMR. Further, utilizing an early multimodal treatment approach has demonstrated improved outcomes for patients with ETMR [4]. Currently, the treatment of ETMR is approached by utilizing a non-selective high dose chemotherapy in combination with a radiotherapy regimen, and in select cases, an autologous stem cell transplantation [6]. Due to the rare occurrences of pediatric cancers, clinical trials involving precision or personalized medicine in this unique population is limited. However, results taken from clinical trials utilizing a precision medicine approach in adult cancer patient populations can be used as examples for how precision medicine is transforming the field of oncology. Schwaederle et al. performed a meta-analysis of 570 phase II studies involving various cancers comparing the outcome end points and overall survival rates between trials that adopted a precision medicine approach. The results from this study highlighted better outcomes for patients who received treatment regimens based upon molecular profiling [7].

Recently, the Children's Oncology Group (COG), in partnership with the National Cancer Institute (NCI), has launched the Pediatric Molecular Analysis for Therapeutic Choice (MATCH) protocol. This protocol entails the implementation of biomarker profiling coupled with single-arm phase II trials of targeted therapies. This initiative represents an important transition for Pediatric Oncology into the field of precision medicine [8].

Development of precision medicine regimens relies heavily on selecting therapeutics based upon the molecular profile of the tumor. Here we report the successful use of molecular profiling and dasatinib therapy with informed parental consent in the multimodal treatment of a 32-month-old patient with ETMR for whom standard therapy had failed.

\section{Case Report}

A 32-month-old, ex-29-week twin B female with no family history of cancer or congenital disease presented to the emergency department with complaints of recurrent headaches. Initial brain computerized tomography (CT) demonstrated a large solid and cystic intra axial tumor with coarse calcifications within the left parietal lobe resulting in significant midline shift. The tumor cyst was treated surgically by placing an Ommaya reservoir to drain cyst volume and relieve symptoms. Biopsy of tumor and cystic fluid allowed for the diagnosis of ETMR-NOS, WHO Grade IV, with extensive necrosis and calcification. The patient was placed on standard induction chemotherapy with each cycle consisting of vincristine $(0.5 \mathrm{mg} / \mathrm{kg} / \mathrm{day}$, weekly), etoposide ( $2.5 \mathrm{mg} / \mathrm{kg}$, days 1 through 3), cyclophosphomaide $(60 \mathrm{mg} / \mathrm{kg}$, days 1 and 2), cisplatin (3.5 mg/kg, day 3). During the third cycle of induction chemotherapy, the patient displayed symptoms consistent with chemotherapy toxicity. Brain magnetic resonance imaging (MRI), with and without contrast, demonstrated an increase in the bulk of the peripheral gyriform enhancing tumor mantel with reaccumulation of the large cystic cavity and progressive disease was noted. Subsequently, induction chemotherapy was discontinued, the solid tumor component was partially resected, and tumor cells studied. 
Chromosome and FISH analysis presented a normal female karyotype with the exception of reported natural variants identified within chromosomes 14, 16, and 22 (data not shown). Patient tumor cells were obtained from excess diagnostic material through an approved study by the Institutional Review Board. Target proteins were subsequently analyzed using XMAP multiplex technology on the Luminex 200 platform coupled with XPONENT 3.1 software according to the manufacturer's suggested protocol. Cell signaling analysis was performed on the partially biopsied tumor which revealed an extensively activated tyrosine kinase signaling molecular profile. Additionally, this tumor was sectioned and imaged by transmission electron microscopy (TEM), as previously described [9]. The signaling profile of the ETMR tumor suggested dasatinib as a potentially viable therapy based upon observed SRC and PDGFR activity (see below). Irradiation therapy was not considered a viable option due to the unwanted side effects of radiation on the developing brain.

A four-month therapy of dasatinib (one $20 \mathrm{mg}$ tablet twice daily) was well-tolerated by the patient. MRI, multiplanar T1, FLAIR, T2, gradient echo and postcontrast diffusion imaging was performed pre- and post-dasatinib treatment to quantitate clinical response. As shown (Fig. 1A, B), the anterior to posterior cystic component was markedly decreased by a $3.46 \mathrm{~cm}$ reduction which also coincided with a $0.3 \mathrm{~cm}$ reduction in size for the solid tumor component. The MRI images of the post operation and 2-year follow up were also found to be clear of visible tumor component (Fig. 1B). The analysis of pre- and post-dasatinib was observed to be molecularly effective in the treatment of this progressive refractory ETMR based on Ki67 proliferation index staining, which displayed decreased proliferation activity (Fig. 2A). Analysis of tumor cell signaling molecules revealed that dasatinib treatment decreased activation of receptor tyrosine kinases PDGFRa and PDGFRb (Fig. 2Bi). Analysis of the activated forms of Src family kinases including SRC, FYN, and LYN were all observed to be significantly reduced (Fig. 2Bii). In addition, activated forms of downstream molecules including STAT1, STAT3, and ERK1/2 were also markedly reduced in response to dasatinib treatment (Fig. 2Biii). Transmission electron microscopy (TEM) of pre- (Fig. 2Ci, Cii) and post dasatinib therapy (Fig. 2Ciii, Civ) illustrated the impact by allowing visualization of increased changes with tumor ultrastructure suggestive of induced cellular autophagy of the ETMR.

In summary of this case, implementing a molecular guided approach in pediatric ETMR allowed for the effective utilization of dasatinib therapy. Dasatinib therapy significantly reduced the brain tumor size allowing for a total resection of the residual tumor (Fig. 1B). An EEG at four months post-surgery demonstrated an unremarkable symmetric profile. Five years post resection, the patient remains disease free, with no evidence of recurrent tumor.

\section{Discussion}

Molecularly targeted therapies have transformed the treatment of cancer [10]; however successful application of pathway-specific inhibitors remains a challenge. The off-label usage of chemotherapeutic agents based on tumor biomarker expression is being heavily investigated. The COG and NCI Pediatric MATCH trial is highly anticipated to advance precision medicine treatment schemes forward in relapsed and refractory pediatric cancer [8]. Currently, at the adult cancer patient level multiple studies have demonstrated the benefit of precision medicine. For example, Tsimberidou et al., investigated the benefit of utilizing molecular profiling to match targeted agents with tumor molecular aberrations in a Phase I Clinical Trials Program. Their study involved the analysis of 1,144 patients, from which 175 were given therapies based upon results identified through molecular profiling. The patients that received a 


\section{Case Reports in Oncology}

precision medicine treatment regimen demonstrated higher overall response rates compared to their counterparts who were given therapies based on standard protocols [11]. More recent success was reported by Kris et al., who demonstrated the potential of multiplex assays in aiding physicians in selecting therapies for treating 275 of 1,007 patients with metastatic lung adenocarcinomas. The results from this study revealed increased survival rates for patients receiving therapies based on multiplex assays [12]. A meta-analysis performed by Fontes Jardim et al. systematically reviewed the results of 112 clinical trials conducted over a tenyear period and compared the efficacy of trials that incorporated a molecular profiling approach. This comprehensive analysis suggested that molecular profiling can be safe and have improved efficacy outcomes with FDA-approved anticancer agents [13]. With the Pediatric MATCH trial underway, it is expected that similar results will be identified in the pediatric cancer population.

It is estimated that 8.2 million individuals die from cancer worldwide and such trends are expected to increase over the next decade [14]. Most patients with metastatic or refractory cancer will exhaust all conventional therapy options. Better understanding of aberrantly regulated signaling networks, at an individualized level, will be critical for developing rational targeted therapies to overcome drug resistance in tumors such as those present in ETMR patients with refractory disease. The results presented herein could aid future ETMR patient stratification schemes that recruit similar molecular activation pathway strategies. Thus, larger clinical studies employing this approach are perhaps warranted.

\section{Acknowledgement}

This work was supported by grants and gifts from the Lizanell and Colbert Coldwell Foundation, Edward N. and Margaret G. Marsh Foundation, The Shiloff Family Foundation, and Grant 5G12MD007592 from the National Institute on Minority Health and Health Disparities, National Institutes of Health.

\section{Statement of Ethics}

The authors have no ethical conflicts to disclose.

\section{Disclosure Statement}

The authors declare that there is no conflict of interest.

\section{References}

1 Louis DN, Perry A, Reifenberger G, von Deimling A, Figarella-Branger D, Cavenee WK, et al. The 2016 World Health Organization Classification of Tumors of the Central Nervous System: a summary. Acta Neuropathol. 2016 Jun;131(6):803-20.

2 Pickles JC, Hawkins C, Pietsch T, Jacques TS. CNS embryonal tumours: WHO 2016 and beyond. Neuropathol Appl Neurobiol. 2018 Feb;44(2):151-62. 
3 Geyer JR, Sposto R, Jennings M, Boyett JM, Axtell RA, Breiger D, et al.; Children's Cancer Group. Multiagent chemotherapy and deferred radiotherapy in infants with malignant brain tumors: a report from the Children's Cancer Group. J Clin Oncol. 2005 Oct;23(30):7621-31.

4 Choi SH, Kim SH, Shim KW, Han JW, Choi J, Kim DS, et al. Treatment Outcome and Prognostic Molecular Markers of Supratentorial Primitive Neuroectodermal Tumors. PLoS One. 2016 Apr;11(4):e0153443.

5 Padovani L, André N, Constine LS, Muracciole X. Neurocognitive function after radiotherapy for paediatric brain tumours. Nat Rev Neurol. 2012 Oct;8(10):578-88.

6 Sung KW, Lim DH, Yi ES, Choi YB, Lee JW, Yoo KH, et al. Tandem High-Dose Chemotherapy and Autologous Stem Cell Transplantation for Atypical Teratoid/Rhabdoid Tumor. Cancer Res Treat. 2016 Oct;48(4):140819.

7 Schwaederle M, Zhao M, Lee JJ, Eggermont AM, Schilsky RL, Mendelsohn J, et al. Impact of Precision Medicine in Diverse Cancers: A Meta-Analysis of Phase II Clinical Trials. J Clin Oncol. 2015 Nov;33(32):3817-25.

8 Allen CE, Laetsch TW, Mody R, Irwin MS, Lim MS, Adamson PC, et al.; Pediatric MATCH Target and Agent Prioritization Committee. Target and Agent Prioritization for the Children's Oncology Group-National Cancer Institute Pediatric MATCH Trial. J Natl Cancer Inst. 2017 May;109(5).

9 Ross JA, Nagy ZS, Kirken RA. The PHB1/2 phosphocomplex is required for mitochondrial homeostasis and survival of human T cells. J Biol Chem. 2008 Feb;283(8):4699-713.

10 Arora A, Scholar EM. Role of tyrosine kinase inhibitors in cancer therapy. J Pharmacol Exp Ther. 2005 Dec;315(3):971-9.

11 Tsimberidou AM, Iskander NG, Hong DS, Wheler JJ, Falchook GS, Fu S, et al. Personalized medicine in a phase I clinical trials program: the MD Anderson Cancer Center initiative. Clin Cancer Res. 2012 Nov;18(22):637383.

12 Kris MG, Johnson BE, Berry LD, Kwiatkowski DJ, Iafrate AJ, Wistuba II, et al. Using multiplexed assays of oncogenic drivers in lung cancers to select targeted drugs. JAMA. 2014 May;311(19):1998-2006.

13 Jardim DL, Schwaederle M, Wei C, Lee JJ, Hong DS, Eggermont AM, et al. Impact of a Biomarker-Based Strategy on Oncology Drug Development: A Meta-analysis of Clinical Trials Leading to FDA Approval. J Natl Cancer Inst. 2015 Sep;107(11):djv253.

14 Ferlay J, Soerjomataram I, Dikshit R, Eser S, Mathers C, Rebelo M, et al. Cancer incidence and mortality worldwide: sources, methods and major patterns in GLOBOCAN 2012. Int J Cancer. 2015 Mar;136(5):E35986. 


\section{Case Reports in Oncology}

\begin{tabular}{l|l}
\hline DOI: $10.1159 / 000497380$ & (c) 2019 The Author(s). Published by S. Karger AG, Basel
\end{tabular} www.karger.com/cro

A

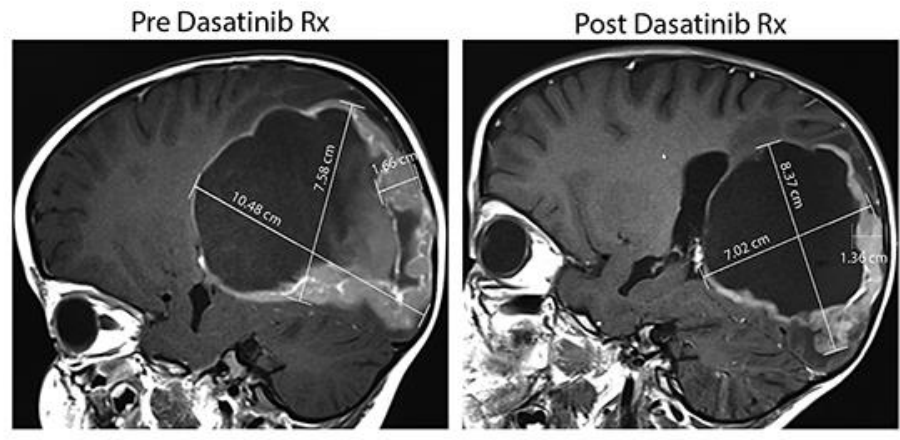

\begin{tabular}{lcrc} 
& Pre Dasatinib & & Post Dasatinib \\
\cline { 2 - 2 } AP Cystic Component & $10.48 \mathrm{~cm}$ & & $7.02 \mathrm{~cm}$ \\
\hline SI Cystic Component & $7.58 \mathrm{~cm}$ & & $8.37 \mathrm{~cm}$ \\
\hline Tumor Component* & $1.66 \mathrm{~cm}$ & & $1.36 \mathrm{~cm}$ \\
\hline
\end{tabular}

B
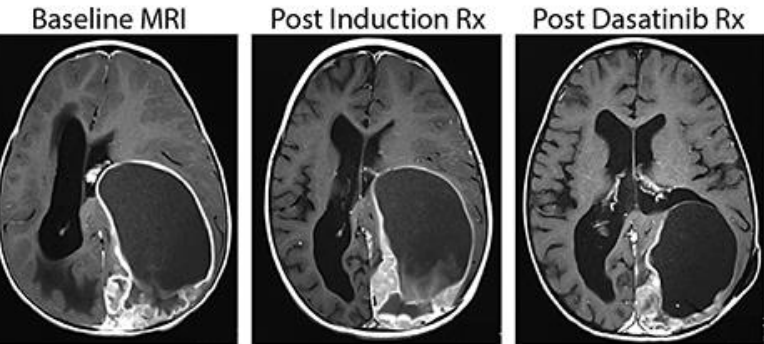

Post Operation
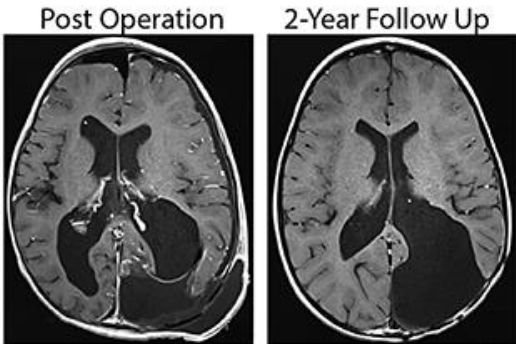

Fig. 1. Brain MRI Demonstrating ETMR Response to Dasatinib Treatment. (A) Cystic component and tumor component measurements demonstrating increased ETMR tumor response to dasatinib treatment. Marked reductions in both cystic and tumor components was noted. (B) Treatment time course demonstrating overall clinical time course. Baseline MRI and Post Induction Treatment (Rx) MRI demonstrated interval increase in bulk of peripheral gyriform enhancing tumor mantle with re-accumulation of the large cystic cavity. Post dasatinib Rx MRI displayed an overall volume reduction of tumor, cystic cavity, and mantle involvement within the parieto-occipital region. Tumor volume reduced from $1.66 \mathrm{~cm}$ to $1.36 \mathrm{~cm}$. (AP: Anterior-Posterior; SI: Superior-Inferior). * Tumor Component measured at widest margin. 


\section{Case Reports in Oncology}

\begin{tabular}{l|l}
\hline Case Rep Oncol 2019;12:211-217 \\
\hline DOI: 10.1159/000497380 & $\begin{array}{l}\text { (c) 2019 The Author(s). Published by S. Karger AG, Basel } \\
\text { www.karger.com/cro }\end{array}$ \\
\hline
\end{tabular}

Hartman et al.: Personalized Molecular Targeted Therapy of ETMR

A

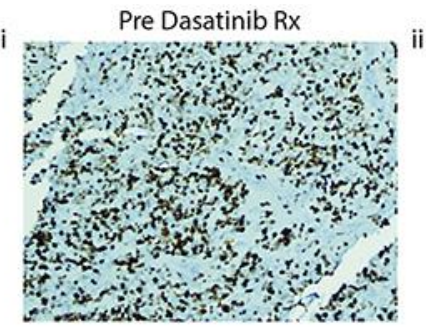

B

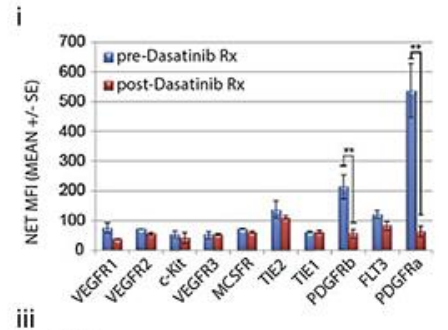

ii
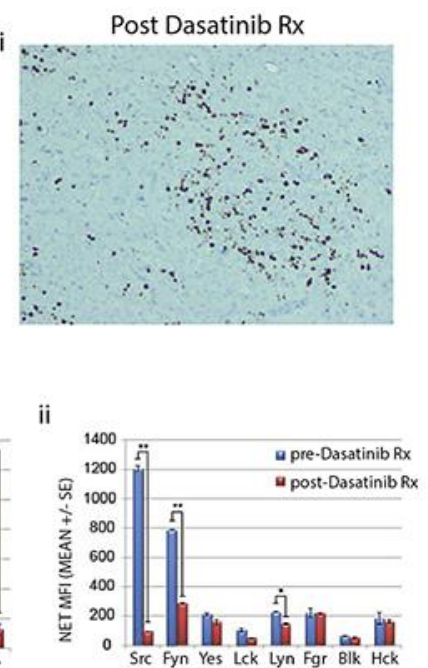

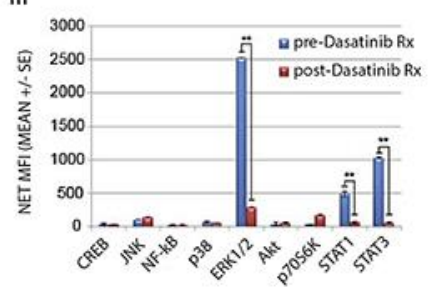

C

Pre Dasatinib Rx

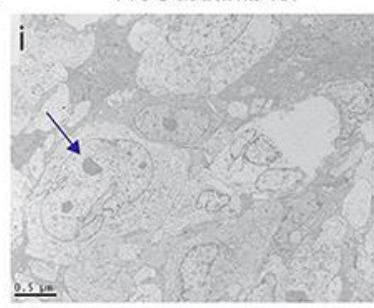

Post Dasatinib Rx
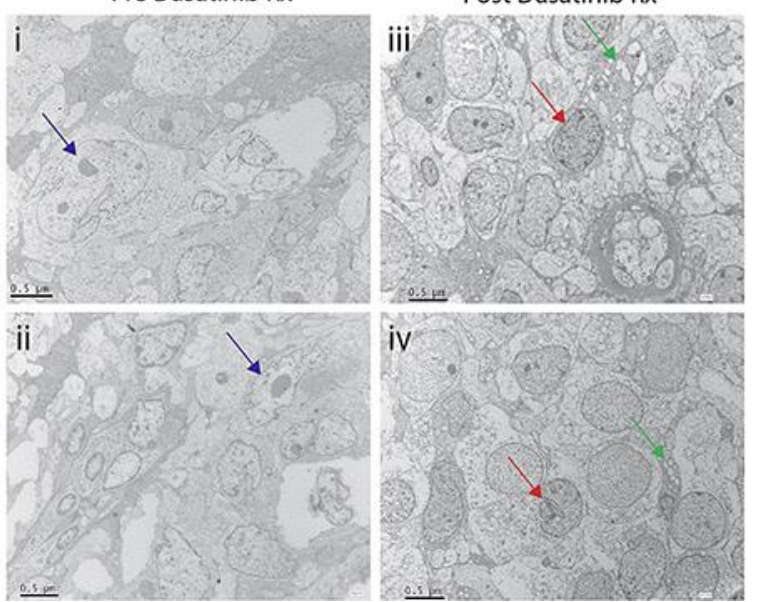

Fig. 2. Patient tumor molecular response and ultrastructural changes to dasatinib treatment. (A) Ki-67 immunopositivity decreased from (i) $50-60 \%$ to (ii) $5 \%$ post-dasatinib treatment. (B) Aberrant activation of (i) RTKs: PDGFRa/b, (ii) SFKs: SRC, FYN, and LYN, and (iii) downstream ERK1/2 and STAT1/3 effectors were significantly reduced in the post-dasatinib Rx tumor. Student's t-tests were employed using SigmaStat3.1 software. $p$ values $<0.05\left(^{*}\right)$ and $<0.01\left(^{* *}\right)$ were considered statistically significant. (C) Ultrastructural examination of ETMR tumor pre- (i, ii) and post-dasatinib Rx (iii, iv). Autophagocytic vacuoles (green arrows) and predominance of nuclear heterochromatin (red arrows) compared to euchromatin (blue arrows) are indicated. 